\title{
Quantum Monte Carlo Study of Electron Correlation in Chromium-Doped Silicon Cluster Cr@ $\mathrm{Si}_{12}$
}

\author{
Kenta Hongo $^{1}$, Vijay Kumar ${ }^{2}$, Yoshiyuki Kawazoe ${ }^{1}$ and Hiroshi Yasuhara ${ }^{1}$ \\ ${ }^{1}$ Institute for Materials Research, Tohoku University, Sendai 980-8577, Japan \\ ${ }^{2}$ Dr. Vijay Kumar Foundation, 45 Bazaar Street, K. K. Nagar (West), Chennai 600 078, India
}

\begin{abstract}
Electron correlation in chromium-doped silicon cluster $\left(\mathrm{Cr} @ \mathrm{Si}_{12}\right)$ in its neutral, positively, and negatively charged states with different nuclear configurations is investigated by means of quantum Monte Carlo methods. It is found that the correlation energy per electron is independent of whether the state is charged or not and about $-1 \mathrm{eV}$ for each of these three states. The total binding energy of the neutral state per atom is $3.5 \mathrm{eV}$, which is divided into the Hartree-Fock contribution nearly equal to $1.2 \mathrm{eV}$ and the correlation contribution as large as $2.3 \mathrm{eV}$. In the Hartree-Fock approximation, the ionization energy is $6.9 \mathrm{eV}$ and the electron affinity is $2.7 \mathrm{eV}$. Correlation increases the ionization energy by $1.7 \mathrm{eV}$ and the electron affinity by $1.4 \mathrm{eV}$. [doi:10.2320/matertrans.47.2617]
\end{abstract}

(Received June 12, 2006; Accepted July 24, 2006; Published November 15, 2006)

Keywords: electron correlation, variational Monte Carlo, diffusion Monte Carlo, doped cluster of silicon

\section{Introduction}

Metal doped silicon clusters are currently attracting great interest as metal encapsulation could offer interesting possibilities of producing size selected silicon clusters in large quantities. Experimentally high abundances of 15 and $16 \mathrm{Si}$ atom clusters doped with a $\mathrm{Cr}, \mathrm{Mo}$, or $\mathrm{W}$ atom were obtained by Beck $^{1)}$ who also speculated that the structure of such clusters could be a cage of $\mathrm{Si}$ and a metal atom at the center. More recently in another experiment ${ }^{2)} 12$ atom silicon cluster doped with a $\mathrm{W}$ atom has been found to be particularly stable and non-reactive with $\mathrm{H}$. It was shown to have a prism structure with $\mathrm{W}$ at the center. Cage clusters of pure silicon are unstable in contrast with carbon clusters. ${ }^{3)}$ Theoretical studies ${ }^{4,5)}$ have shown that silicon clusters $\left(\mathrm{Si}_{n}\right)$ with high symmetries are stabilized by doping a transition metal (TM) atom such as $\mathrm{Ti}$ and $\mathrm{Zr}$. Further theoretical and experimental studies on metal doped silicon cage clusters $\left(\mathrm{TM} @ \mathrm{Si}_{n}\right.$ ) have been widely performed ${ }^{6-11)}$ and the predictions of a high symmetry Ti@ $\mathrm{Si}_{16}$ cluster have been confirmed ${ }^{12)}$ with nearly exclusive abundance of this cluster.

Very recently, Kawamura et al. ${ }^{10,11)}$ have performed a systematic study of magic behavior of the chromium-doped silicon clusters $\left(\mathrm{Cr} @ \mathrm{Si}_{n}\right)$ in the framework of density functional theory (DFT) ${ }^{13,14)}$ using the generalized gradient approximation (GGA) ${ }^{15)}$ and the hybrid exchange-correlation functional (B3PW91). ${ }^{16)}$ They have found that $\mathrm{Cr} @ \mathrm{Si}_{12}$ and $\mathrm{Cr} @ \mathrm{Si}_{15}$ calculated with GGA show magic behaviors, but the use of B3PW91 makes the magic behavior of $\mathrm{Cr} @ \mathrm{Si}_{15}$ very poor. Therefore they have arrived at the conclusion that the accurate treatment of exchange and correlation is needed for the investigation of the bonding nature of these clusters.

In the present paper, we apply quantum Monte Carlo (QMC) methods such as variational Monte Carlo (VMC) and diffusion Monte Carlo (DMC) methods ${ }^{17)}$ to the quantitative study of $\mathrm{Cr} @ \mathrm{Si}_{12}$ clusters in its neutral, positively, and negatively charged states. VMC and DMC both can provide an accurate evaluation of the ground-state energy of the system, which is comparable to the conventional quantum chemistry methods such as configuration interaction (CI) method. The computational cost of QMC is nearly proportional to $N^{3}$, while that of CI at least to $N^{6}$ where $N$ is the number of electrons in the system. The QMC algorithm is appropriate for parallel computing. Hence we have to adopt QMC methods for the quantitative study of large systems instead of a so-called truncated CI method involving the sizeconsistency problem. The use of VMC and DMC enables one to investigate the role of correlation in the bonding nature of clusters.

Section II deals with the computational methods. Section III provides numerical results for various quantities together with some discussions.

\section{Computational Methods}

VMC is a method of evaluating the expectation value of an operator with respect to a trial wavefunction $\Psi_{T}$ through Monte Carlo integration. A typical trial wavefunction adopted for VMC usually contains a number of parameters whose values are optimized such that the square of the standard deviation for the VMC total energy (i.e., variance $\sigma^{2}$ ) takes its minimum. ${ }^{18,19)}$ In order to approximate the variance to its smallest possible value equivalent to zero, one must pay attention to the singular behavior of the exact manyelectron wavefunction $\Phi\left(\left\{\boldsymbol{r}_{i}\right\},\left\{\boldsymbol{r}_{I}\right\}\right)$ caused by the shortdistance behavior of the Coulomb interaction. Consider $\Phi\left(\left\{\boldsymbol{r}_{i}\right\},\left\{\boldsymbol{r}_{I}\right\}\right)$ where $\left\{\boldsymbol{r}_{i}\right\}$ and $\left\{\boldsymbol{r}_{I}\right\}$ denote a set of electronic coordinates and a set of nuclear coordinates, respectively. In general $\Phi\left(\left\{\boldsymbol{r}_{i}\right\},\left\{\boldsymbol{r}_{I}\right\}\right)$ exhibits a discontinuity in its first-order derivative as $\boldsymbol{r}_{i} \rightarrow \boldsymbol{r}_{I}$ or $\boldsymbol{r}_{i} \rightarrow \boldsymbol{r}_{j}$, i.e., whenever an electron approaches to a nucleus or another electron. In either limit $\Phi\left(\left\{\boldsymbol{r}_{i}\right\},\left\{\boldsymbol{r}_{I}\right\}\right)$ remains finite but exhibits a cusp-like singular behavior with respect to the nucleus-electron separation $\mid \boldsymbol{r}_{i}-$ $\boldsymbol{r}_{I} \mid$ or the electron-electron separation $\left|\boldsymbol{r}_{i}-\boldsymbol{r}_{j}\right| .^{20)}$ The accurate description of all cusp discontinuities in the trial wavefunction is indispensable to make the variance convergent. The point is how to approximate the variance to its smallest possible value by taking full account of those cusps in the wavefunction. 
The trial wave function we have adopted in the present study is of the Slater-Jastrow type:

$$
\Psi_{T}\left(\left\{\boldsymbol{r}_{i}\right\},\left\{\boldsymbol{r}_{I}\right\}\right)=D\left(\left\{\boldsymbol{r}_{i}\right\},\left\{\boldsymbol{r}_{I}\right\}\right) \exp \left[J\left(\left\{\boldsymbol{r}_{i}\right\},\left\{\boldsymbol{r}_{I}\right\}\right)\right],
$$

where $D$ and $\exp [J]$ denote a Slater determinant and a Jastrow factor, respectively. For each of the three clusters, we have used the same nuclear configuration $\left\{\boldsymbol{r}_{I}\right\}$ that Kawamura et al. ${ }^{10,11)}$ obtained from their DFT study. The neutral and anion clusters have both the same hexagonal prism structure with $D_{6 h}$ symmetry, while the cation cluster has a structure distorted from $D_{6 h}$ to $C_{2 v}$ symmetry. We have calculated the one-electron orbitals entering the determinant $D$ with Gaussian 98 code, 6-311+G* $\mathrm{G}^{*}$ basis set, and B3PW91. ${ }^{21)}$ These orbitals give no appropriate description of the nucleuselectron cusp since the Gaussian code is employed. In order to recover the cusp behavior we have adopted an extrapolation method in which the main-body behavior of these orbitals is smoothly connected to an appropriate analytic form near the nuclei. ${ }^{22}$ ) We have adopted the Jastrow function $J$ constructed from three terms: The first is the homogeneous isotropic electron-electron term, the second the isotropic electron-nucleus term centered on the nuclei, and the third the isotropic electron-electron-nucleus term centered on the nuclei. ${ }^{23,24)}$ The first is responsible for the electron-electron cusp for both spin-antiparallel and spinparallel configurations.

DMC is a technique for numerically solving the manyelectron Schödinger equation for any stationary state. The time-dependent Schrödinger equation involves an exponential factor with an imaginary argument $\exp (-i \hat{H} t / \hbar)$, where $\hat{H}$ is the many-electron Hamiltonian. By introducing the imaginary-time $\tau=i t$, the equation can be transformed into a diffusion-type Fokker-Planck equation. Its imaginary-time evolution, owing to the presence of a damping factor $\exp (-\hat{H} \tau / \hbar)$, can in principle lead to the exact wavefunction after a long enough interval $\tau$, unless the starting trial wavefunction is completely orthogonal to the exact one.

We have used in DMC the same starting trial wavefunction as in VMC. It is evident that the accuracy of DMC depends critically on the starting trial wavefunction as follows:

(1) DMC usually assumes the fixed-node approximation in which the nodal surfaces of the DMC wavefunction are assumed to be the same as those of the VMC wavefunction. ${ }^{25,26)}$

(2) The lower starting energy leads to the more rapid convergence of the final DMC energy.
(3) The lower deviation of the VMC wavefunction from the final DMC wavefunction leads to the more numerically stable results of DMC.

The number of parameters in the Jastrow factor is totally 73 for each atom and 157 for each cluster in the present study. These parameters are optimized by minimizing the variance of the VMC energy:

$$
\sigma^{2}=\frac{\left\langle\Psi_{T}\left|\hat{H}^{2}\right| \Psi_{T}\right\rangle}{\left\langle\Psi_{T} \mid \Psi_{T}\right\rangle}-E_{\mathrm{VMC}}^{2},
$$

where $E_{\mathrm{VMC}}$ denotes the VMC energy. ${ }^{18,26)}$ We have also performed the VMC calculation omitting the Jastrow factor to evaluate the Hartree-Fock energy. To attain reasonably converged results we have accumulated the numerical results over $8 \times 10^{7}-1 \times 10^{8}$ steps for the HF calculations, over $3 \times$ $10^{8}-7 \times 10^{8}$ steps for the VMC calculations, and over $1 \times$ $10^{6}-3 \times 10^{6}$ steps for the DMC calculations. In the DMC calculations for the atoms and clusters we have used a time step of 0.0005 a.u., which is small enough to make the timestep error negligibly small. We have performed all the HF, VMC, and DMC calculations using the CASINO code. ${ }^{27)}$

\section{Results and Discussions}

Table 1 gives a list of QMC values for the ground-state energy of the $\mathrm{Si}$ atom, the $\mathrm{Cr}$ atom, and the $\mathrm{Cr} @ \mathrm{Si}_{12}$ cluster in its neutral, positively, and negatively charged states. $E_{\mathrm{HF}}$ denotes the energy calculated with the Slater determinant alone. $E_{\mathrm{VMC}}$ denotes the VMC energy calculated with the Slater-Jastrow wavefunction. $E_{\mathrm{DMC}}$ denotes the DMC energy. The ratio $\eta$ is defined as

$$
\eta=\frac{E_{\mathrm{HF}}-E_{\mathrm{VMC}}}{E_{\mathrm{HF}}-E_{\mathrm{DMC}}} \times 100 \% .
$$

The ratio $\eta$ gives a measure of how the quality of the starting trial wavefunction adopted in VMC is appropriate for DMC. The variance for the $\mathrm{HF}$ calculation is denoted by $\sigma_{\mathrm{HF}}^{2}$ and that for the VMC calculation by $\sigma_{\mathrm{VMC}}^{2}$. The values of $\sigma_{\mathrm{HF}}^{2}$ and $\sigma_{\mathrm{VMC}}^{2}$ are also listed in Table 1 .

The higher value of $\eta$ and the lower value of $\sigma_{\mathrm{VMC}}^{2}$ lead to the more rapid convergence of the DMC energy. The present study for the atoms and clusters gives the value of $\eta$ that is comparable with the previously reported one. ${ }^{28)}$ The variance $\sigma_{\mathrm{HF}}^{2}$ is larger than the variance $\sigma_{\mathrm{VMC}}^{2}$ by a factor of 2.9-3.1 for the atoms and by a factor of 1.5-3.6 for the clusters. The smaller value of $\sigma_{\mathrm{VMC}}^{2}$ means that the use of the Jastrow

Table 1 A list of QMC valeus for the ground-state energy of the $\mathrm{Si}$ atom, the $\mathrm{Cr}$ atom, and the Cr@ $\mathrm{Si}_{12}$ clusters. The ratio $\eta$ and the variance $\sigma^{2}$ in the HF and VMC calculations are also given. Statistical errors in QMC are indicated in the parenthesis. All energies are in hartree units.

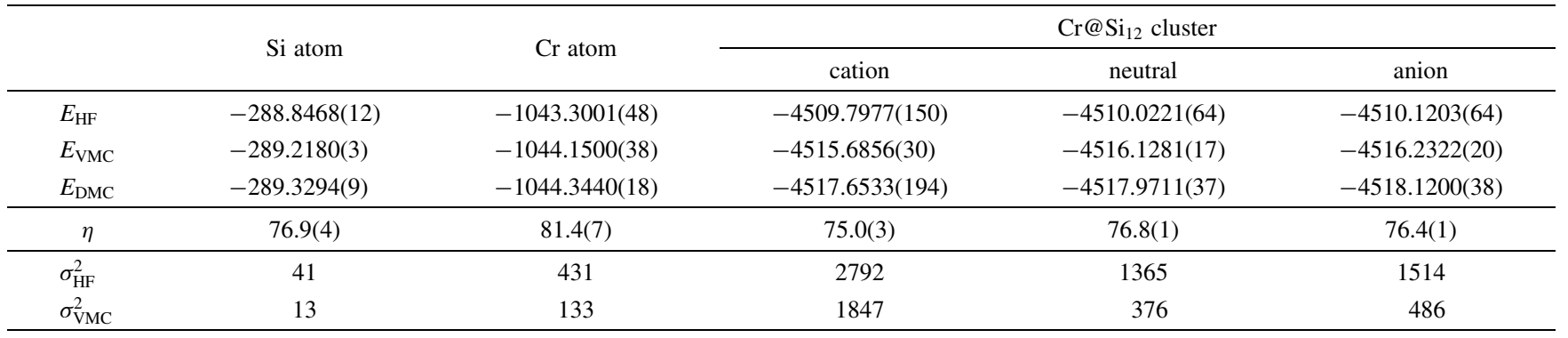


Table 2 A list of the correlation energy per electron $\varepsilon_{c}$ of the $\mathrm{Si}$ atom, the $\mathrm{Cr}$ atom, and the $\mathrm{Cr} @ \mathrm{Si}_{12}$ clusters. Statistical errors in QMC are indicated in the parenthesis. All energies are in $\mathrm{eV}$ units.

\begin{tabular}{llllcl}
\hline & \multirow{2}{*}{ Si atom } & Cr atom & \multicolumn{3}{c}{$\mathrm{Cr} @ \mathrm{Si}_{12}$ cluster } \\
\cline { 3 - 6 } & & & cation & neutral & anion \\
\hline$\varepsilon_{c}^{\mathrm{VMC}}$ & $-0.721(2)$ & $-0.963(7)$ & $-0.838(2)$ & $-0.865(1)$ & $-0.861(1)$ \\
$\varepsilon_{c}^{\mathrm{DMC}}$ & $-0.938(3)$ & $-1.183(6)$ & $-1.119(3)$ & $-1.126(1)$ & $-1.127(1)$ \\
$\varepsilon_{c}^{\mathrm{SDCI}}$ & -0.120 & -0.091 & & & \\
\hline
\end{tabular}

Table 3 A list of QMC values for the binding energy per atom (BE), the ionization energy (IE), and the electron affinity (EA) for the neutral cluster. Statistical errors in QMC are indicated in the parenthesis. All energies are in $\mathrm{eV}$ units.

\begin{tabular}{llcl}
\hline & HF & VMC & DMC \\
\hline BE & $1.17(4)$ & $2.85(1)$ & $3.50(2)$ \\
IE & $6.9(3)$ & $12.0(1)$ & $8.6(5)$ \\
EA & $2.7(1)$ & $2.8(1)$ & $4.1(1)$ \\
\hline
\end{tabular}

factor successfully describes electron-electron cusps. The variance $\sigma_{\mathrm{VMC}}^{2}$ for the anion cluster is slightly larger than that for the neutral cluster. On the other hand, the variance $\sigma_{\mathrm{VMC}}^{2}$ for the cation cluster is by a factor of 4.9 larger than that for the neutral cluster. The statistical error in the DMC energy calculations of the cation cluster is largest. This is probably ascribed to the fact that the cation cluster $\left(C_{2 v}\right)$ has lower symmetry than the neutral cluster $\left(D_{6 h}\right)$.

Table 2 shows a list of the correlation energy per electron $\varepsilon_{c}$ of the $\mathrm{Si}$ atom, the $\mathrm{Cr}$ atom, and the neutral, positively, and negatively charged $\mathrm{Cr} @ \mathrm{Si}_{12}$ clusters in VMC and DMC. The correlation energy is defined as the difference between the exact ground-state energy and the HF energy. For comparison we also list the SDCI correlation energy $\varepsilon_{c}$ of the Si and $\mathrm{Cr}$ atoms, which we have evaluated by the same GAUSSIAN 98 code and basis set $6-311+\mathrm{G}^{*}$ as used in the QMC calculations. We have attempted to perform the same SDCI calculations for the clusters, but arrived at no reasonably converged results.

The correlation energy per electron $\varepsilon_{c}$ is of the same order common to atoms, molecules, and solids. It is approximately $-1 \mathrm{eV}$. This means that the magnitude of $\varepsilon_{c}$ is rather insensitive to both the number of electrons in the system and the electronic structure. According to VMC and DMC, the magnitude of $\varepsilon_{c}$ for the $\mathrm{Si}$ atom is smaller than that for the $\mathrm{Cr}$ atom. However, SDCI fails to give the correct order of $\varepsilon_{c}$ for the two atoms. This is because SDCI is not size-consistent. The correlation energy per electron in DMC is about $-1 \mathrm{eV}$ for the neutral cluster, its cation and anion. From the above numerical results it is evident that DMC has the property of size-consistency in the evaluation of the correlation energy.

Table 3 gives a list of QMC values for the binding energy per atom (BE), the ionization energy (IE), and the electron affinity (EA) for the neutral cluster. The total binding energy of the neutral state per atom is $3.5 \mathrm{eV}$, which is divided into the HF contribution nearly equal to $1.2 \mathrm{eV}$ and the correlation contribution as large as $2.3 \mathrm{eV}$. The ionization energy or the electron affinity is defined as the total energy difference between the neutral state and positively or negatively charged state with different nuclear configurations. Note that correlation increases the ionization energy by $1.7 \mathrm{eV}$ and the electron affinity by $1.4 \mathrm{eV}$.

\section{Acknowledgments}

The authors' calculations were performed using the facilities of the Center for Computational Materials Science (IMR; Institute for Materials Research, Tohoku University) and the Numerical Materials Simulator of the Computational Materials Science Center (NIMS; National Institute for Materials Science). The authors would like to thank Dr. Ryo Maezono for the generous provision of computational facilities.

\section{REFERENCES}

1) S. M. Beck: J. Chem. Phys. 90 (1989) 6306.

2) H. Hiura, T. Miyazaki and T. Kanayama: Phys. Rev. Lett. 86 (2001) 1733.

3) M. Broyer, M. Pellarin, B. Baguenard, J. Lermé, J. L. Vialle, P. Melinon, J. Tuaillon, V. Dupuis, B. Prevel and A. Perez: Cluster Assembled Materials, ed. by K. Sattler [Mater. Sci. Forum 232 (1996) 27].

4) V. Kumar and Y. Kawazoe: Phys. Rev. Lett. 87 (2001) 045503.

5) V. Kumar and Y. Kawazoe: Phys. Rev. B 65 (2002) 073404.

6) S. N. Khanna, B. K. Rao and P. Jena: Phys. Rev. Lett. 89 (2002) 016803.

7) M. Ohara, K. Koyasu, A. Nakajima and K. Kaya: Chem. Phys. Lett. 371 (2003) 490.

8) F. Hagelberg, C. Xiao and W. A. Lester, Jr.: Phys. Rev. B 67 (2003) 035426.

9) P. Sen and L. Mitas: Phys. Rev. B 68 (2003) 155404.

10) H. Kawamura, V. Kumar and Y. Kawazoe: Mater. Trans. 45 (2004) 1429.

11) H. Kawamura, V. Kumar and Y. Kawazoe: Phys. Rev. B 70 (2004) 245433.

12) K. Koyasu, M. Akutsu, M. Mitsui and A. Nakajima: J. Am. Chem. Soc. 127 (2005) 4998.

13) P. Hohenberg and W. Kohn: Phys. Rev. 136 (1964) B864.

14) W. Kohn and L. J. Sham: Phys. Rev. 140 (1965) A1133.

15) J. P. Perdew: Electronic Structure of Solids '91, ed. by P. Ziesche and H. Eschrig (Akademie Verlag, Berlin, 1991).

16) A. D. Becke: J. Chem. Phys. 98 (1993) 5648.

17) W. M. C. Foulkes, L. Mitas, R. J. Needs and G. Rajagopal: Rev. Mod. Phys. 73 (2001) 33.

18) C. J. Umrigar, K. G. Wilson and J. W. Wilkins: Phys. Rev. Lett. 60 (1988) 1719

19) P. R. C. Kent, F. J. Needs and G. Rajagopal: Phys. Rev. B 59 (1999) 12344.

20) T. Kato: Commun. Pure Appl. Math. 10 (1957) 151.

21) M. J. Frisch et al.: GAUSSIAN 98, Revision A.11.1, (Gaussian Inc., Pittsburgh, PA) (2001).

22) A. Ma, M. D. Towler, N. D. Drummond and R. J. Needs: J. Chem. Phys. 122 (2005) 224322.

23) R. J. Jastrow: Phys. Rev. 98 (1955) 1479.

24) N. D. Dorummond, M. D. Towler and R. J. Needs: Phys. Rev. B 70 (2004) 235119.

25) J. B. Anderson: J. Chem. Phys. 65 (1976) 4121.

26) P. J. Reynolds, D. M. Ceperley, B. J. Alder and W. A. Lester, Jr.: J. Chem. Phys. 77 (1982) 5593.

27) R. J. Needs, M. D. Towler, N. D. Drummond and P. R. C. Kent: CASINO version 1.7 User Manual, (University of Cambridge, Cambridge, 2004).

28) T. Oyamada, K. Hongo, Y. Kawazoe and H. Yasuhara: J. Chem. Phys. 125 (2006) 014101. 